\title{
Characterization and regulation in the expression of a gene coding for the intermediate filament protein desmin
}

(synthetic oligonucleotide probes/muscle differentiation/vimentin)

\author{
Yassemi G. Capetanaki, John Ngai, and Elias LaZarides \\ Division of Biology, California Institute of Technology, Pasadena, CA 91125 \\ Communicated by Giuseppe Attardi, July 12, 1984
}

\begin{abstract}
Using synthetic oligonucleotide probes, we have isolated chicken cDNA clones for the intermediate filament protein desmin. We show that the gene for this protein probably exists as a single copy in the haploid chicken genome and is transcribed into one mature mRNA species of $\approx 2.4$ kilobases. Expression of this mRNA is tissue-specific, as it is present in high abundance in smooth and skeletal muscle but is absent from erythrocytes, spinal cord, and lens cells. A 10- to 20-fold increase in desmin mRNA is observed in myogenic cells upon fusion, which suggests that the level of expression of the desmin gene and the accumulation of desmin filaments during muscle differentiation is regulated at the transcriptional and/ or posttranscriptional level but not at the translational level. Hybridization studies and nucleotide sequence comparison of the cDNAs specific for desmin and for other intermediate filament subunits reveal a region that is highly conserved $(80 \%)$ among different members of the intermediate filament protein gene superfamily, with the exception of the chicken vimentin gene; this gene appears to be less homologous to the genes for the other chicken intermediate filament subunits than the mammalian vimentin gene is to the genes for other mammalian intermediate filament proteins and to the chicken desmin gene.
\end{abstract}

Of the various intermediate filament subunits, desmin exhibits one of the highest degrees of tissue specificity in its expression since it is expressed predominantly by skeletal, cardiac, and most types of smooth muscle (for review, see ref. 1). Desmin accumulates in committed myogenic cells, with a concomitant accumulation of desmin-containing intermediate filaments $(2,3)$. In addition, desmin-containing intermediate filaments exhibit a rearrangement in their cytoplasmic distribution in cultured myogenic cells: the cytoplasmic filament-type distribution is replaced by a sarcomeric $\mathrm{Z}$-line association $(2,3)$. This rearrangement is presumed to reflect a function of these filaments in muscle morphogenesis, and this supposition is supported by the observation that, in adult striated muscle, desmin is associated with the peripheries of $\mathrm{Z}$ discs, where it forms a network extending along the lateral axis of the muscle fiber $(1,4,5)$.

To gain insight into the molecular mechanisms responsible for the involvement of desmin in muscle morphogenesis and to further understand the tissue-specific expression not only of this molecule but of all intermediate filament proteins, we have isolated desmin cDNA clones and used them to show that the desmin gene probably exists as a single copy in the chicken genome and is transcribed into one mRNA species that is predominantly expressed in muscle cells. We show that the accumulation of desmin filaments during terminal differentiation of muscle cells is regulated mainly by the abundance of desmin mRNA. On the other hand, the regulation of the morphogenetic redistribution of desmin filaments

The publication costs of this article were defrayed in part by page charge payment. This article must therefore be hereby marked "advertisement" in accordance with 18 U.S.C. $\$ 1734$ solely to indicate this fact. to the peripheries of $\mathrm{Z}$ discs appears to be mainly posttranslational.

\section{MATERIALS AND METHODS}

Synthesis of the Oligonucleotide Probes. Two mixed oligonucleotide probes were synthesized. The first one, a mixture of 32 heptadecamers, 3'-G-T-(C/T)-G-T-(C/T)-G-T-(C/T)G-T-(G/A)-C-T-(C/T)-C-A-5', is derived from the amino acid sequence Gln-Gin-Gin-His-Glu-Val at the carboxyl terminus of desmin $(6,7)$ and is specific for desmin (referred to as the $\mathrm{D}$ probe). The second one, a family of 16 tetradecamers, 3'-T-T-(G/A)-C-A-(G/C/A/T)-T-T-(C/T)-T-A-C-CG-5', is derived from the amino acid sequence Asn-Val-LysMet-Ala located 76 amino acids from the carboxyl terminus and is common to both desmin and vimentin (referred to as the DV probe). Both probes were synthesized by the solidphase phosphotriester method (8).

Construction and Screening of the Gizzard cDNA Library Using the Synthetic Probes. Poly $(\mathrm{A})^{+}$RNA from 1-week-old chick gizzards was enriched in desmin mRNA by size fractionation on sucrose gradients (9) and used to construct a cDNA library (10). Prehybridizations, hybridizations, and washes of colony filters were carried out as described (10). The hybridization and final wash temperatures were $38^{\circ} \mathrm{C}$ in the case of the heptadecamer and $25^{\circ} \mathrm{C}$ in the case of the tetradecamer.

RNA and DNA Blot-Hybridization. RNA blots were performed as described (11) with some modifications (10), using the transfer system described by Southern (12). Genomic DNA blots were performed using the Southern procedure (12) as modified (13). Hybridizations of ${ }^{32} \mathrm{P}$-labeled nicktranslated probes at $\approx 5 \times 10^{5} \mathrm{cpm}$ - or $2 \times 10^{6} \mathrm{cpm} / \mathrm{ml}$ to filter-bound RNA or DNA, respectively, were done as described (13) at $42^{\circ} \mathrm{C}$ for either $15 \mathrm{hr}$ (RNA blots) or $40 \mathrm{hr}$ (DNA blots). Washing conditions were similar to those described (10).

\section{RESULTS}

Isolation and Characterization of Desmin cDNA Clones Using Synthetic Oligonucleotide Probes. Desmin cDNA clones were isolated from a chicken gizzard cDNA library screened with the two synthetic oligonucleotide probes deduced from the amino acid sequence of two distinct regions of the desmin polypeptide (6) (see Materials and Methods). The DV probe is the one we have used previously to isolate the vimentin gene by screening a chicken genomic library (10). The specificity of the probes was tested by RNA blot hybridization experiments. Blots of gizzard poly $(A)^{+}$RNA, which contains negligible quantities of vimentin mRNA, revealed the same single mRNA band of $\approx 2.4$ kilobases $(\mathrm{kb})$ with either of the two probes (results not shown), suggesting that both probes predominantly detect desmin sequences in gizzard RNA.

Abbreviations: kb, kilobase(s); GFAP, glial fibrillary acidic protein. 


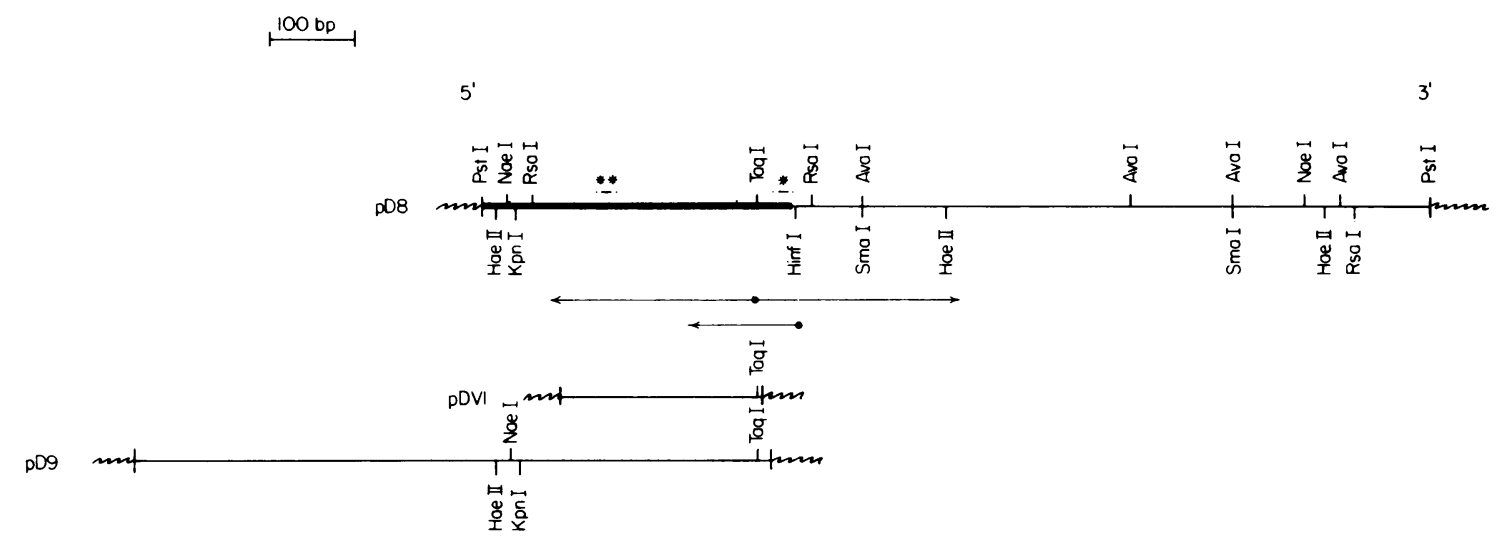

FIG. 1. Restriction map analysis of desmin cDNA insert. The coding region is designated by the thick line and the 3 ' noncoding region by the thin line. The orientation of transcription and the end of the coding region was first estimated by the relative positions of hybridization of the synthetic probes, $\mathrm{D}(*)$ and $\mathrm{DV}(* *)$, and subsequently determined precisely by nucleotide sequencing (see Fig. 2 ). The alignment of clones pDV1 and pD9 relative to pD8 is also shown. The horizontal arrows under pD8 indicate the extent and direction of sequencing of this clone (Fig. 2). bp, Base pairs.

Of $\approx 23,000$ clones screened, none was positive with both probes, though some were later found to contain both regions. About 34 positive clones were obtained with the $\mathrm{D}$ probe and 5 were obtained with the DV probe. All these clones were further analyzed by RNA blots. Clones from pools that gave RNA bands of the expected molecular weight were subjected to positive hybrid-selected translation. The in vitro translation product from RNA selected by pDV1, one of the cDNA plasmids that was detected initially with the DV probe, was identified as desmin by immunoprecipitation with antibodies specific for chicken desmin (data not shown). Thus, this clone was tentatively identified as desmin-specific. Further screening of the gizzard cDNA library with the insert of pDV1 identified nine additional potential desmin clones. Fig. 1 shows the restriction map analysis of one of the isolated desmin recombinants, pD8. The orientation of transcription and the exact positions of the synthetic probe sequences were confirmed by partial sequencing of pD8 (Fig. 2). The identity of the deduced amino acid sequence with the primary sequence of chicken gizzard desmin (6) unequivocally confirmed pD8 as a desmin cDNA. The sequenced segment of the pD8 clone spans 494 bases of desmin mRNA encoding the last 98 amino acids of the carboxyl terminus and including 197 bases of $3^{\prime}$ untranslated sequence (Fig. 2). Comparison of the $3^{\prime}$ noncoding sequence with that of chicken vimentin cDNA (15) reveals no homology. A peculiar feature of this $3^{\prime}$ untranslated region of desmin mRNA is its $77 \%(\mathrm{G}+\mathrm{C})$ content.

Distribution of the Desmin-Specific Sequences in the Chicken Genome. Preliminary restriction map analysis of all the isolated desmin cDNAs showed that all of them have identical internal restriction fragments, suggesting that all are encoded by the same gene. To obtain some information concerning the distribution of desmin-specific sequences in the chicken genome, we carried out genomic DNA blot analyses. Initially, we used as probes either the small (270-basepair insert) cDNA plasmid pDV1 or the entire pD8. The inserts of these plasmids, or the whole plasmids, were nicktranslated and hybridized to DNA blots of BamHI-, HindIII-, EcoRI-, and Sac I-restricted chicken genomic DNA. As shown in Fig. 3, multiple bands were observed in all cases. Of the three to five bands observed, at least two or three hybridized more intensely. Hybridization with pDV1 gave a pattern qualitatively similar to the pattern obtained with pD8, except that the differences in the intensities of the various bands were much smaller than in the case of pD8 (data not shown). Incomplete digestion of the DNA was excluded since the same DNA blots probed with a vimentin genomic clone (Fig. 3) gave the expected single band for all the digests (15).
5. CCA CAC CTG AaG GaT GAG ATC GCC CGG CAC CTC CGC GAG TAC CAG GAC CTG CTC AAT GTC Arg His Leu Lys Asp Glu Met Ala Arg His Leu Arg Glu Tyr Gln Asp Leu Leu Asn Val 10 AAG ATG GCC TTG GAC GTG GAG ATC GCC ACC TAC CGC AAG CTC CTC GAG GGC GAC GAC AAC Lys Met Ala Leu Asp Val Glu Ile Ala Thr Tyr Arg Lys Leu Leu Glu Gly Glu Glu Asn 30

$$
150
$$

150
CGC ATC AGC ATC CCC ATC CAC CAG ACC TTT GCC TCT GCT CTC AAT TTC CGA GAG ACC AGC Arg Ile Ser Ile Pro Met His Gln Thr Phe Ala Ser Ala Leu Asn Phe Arg Glu Thr Ser 210
CCA GAC CAG CGT GCC TCC GAG GTG CAC ACC AAG AAC ACA GTC ATC ATC AAA ACC ATC GAA Pro Asp Clh Arg Gly Ser Glu Val His Thr Lys Lys Thr Val Met Ile Lys Thr Ile Glu

70

270 ACT CGT GAT GGA GAG GTG GTC AGC GAG GCG ACC CAG CAG CAC CAC GAG GTC CTC TAG AAG Thr Arg Asp Gly Glu Val Val Ser Glu Ala Thr Gln Gln Gln His Glu Val Leu Stp 90

ACTCTGTCCCGG TGCCCACCCGCCCTTCTGCCCCCCCCAAGCCCGTACTCCCCCCGACACGGCCGCCTCCGGGCACGG CCCCCTCCCCCCCCACCCCCGGCCTCTCTCCTTCGCCTTCGCAGCCTTCCCCCTAGCGCAAGACCAGGGCCCGGCCCC GGGGCGCCAGACAGGGACGGCGGGCACAGCCGGCAAGG
Fig. 2. Partial nucleotide sequence of the desmin pD8 insert. A segment of 494 nucleotides from pD8 was sequenced by the MaxamGilbert technique (14). It includes the region encoding the 98 amino acids at the carboxyl-terminal end and 197 bases of the $3^{\prime}$ untranslated region. The predicted amino acid sequence is shown below the corresponding DNA sequence. The sequences corresponding to the synthetic oligonucleotide probes used to isolate the desmin cDNA clones are in boxes. Stp, stop codon of the reading frame. 


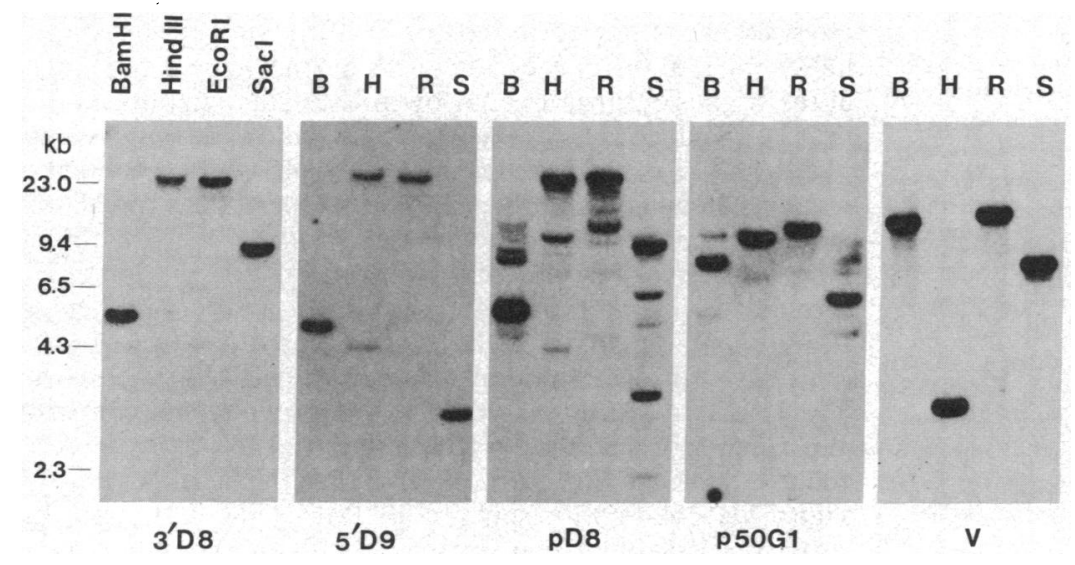

FIG. 3. Distribution of the desmin-specific sequences in the chicken genome. High molecular weight chicken DNA $(3 \mu \mathrm{g})$ was digested with BamHI (B), HindIII (H), EcoRI (R), and Sac I (S), and the resulting fragments were separated on $0.9 \%$ agarose gels and transferred to nitrocellulose. The DNA on the filters was then hybridized to the following nick-translated probes: the $3^{\prime} \mathrm{D} 8$ fragment of the $\mathrm{pD} 8$ desmin cDNA clone containing all the Ava I fragments of the 3' untranslated region; the 5'D9 fragment of the pD9 desmin CDNA, derived by digestion with Nae I (see Fig. 1) and lacking the conserved region that is included in the pDV1 clone; the pD8 cDNA; the p50G1 cDNA; and part of the chicken vimentin genomic clone $\mathrm{V}(10)$.
pDV1 sequences correspond to a conserved stretch of $\approx 40$ amino acids comprising the $\mathrm{COOH}$-terminal portion of the rod domain of the protein (7), adjacent to the beginning of the variable carboxyl terminus. Consequently, this DNA probe and $\mathrm{pD} 8$, both of which contain these sequences (see Fig. 1), could cross-react with other intermediate filament protein genes; this cross-reaction was not expected to occur with the vimentin gene, since pDV1 and pD8 do not crossreact with vimentin mRNA in RNA blots (see below) and genomic blots with corresponding vimentin gene sequences give only single bands (Fig. 3). Further genomic blot analysis showed that cross-hybridization with other members of the intermediate filament protein gene superfamily indeed occurs. When genomic blots were hybridized with sequences corresponding to the $3^{\prime}$ untranslated region of the desmin mRNA (3'D8, which consists of all the Ava I fragments of the $3^{\prime}$ noncoding region of $\mathrm{pD} 8$ isolated from a gel), only single bands were observed in all restriction digests, which corresponded to the strongest bands obtained with pD8 (Fig. 3). Furthermore, when similar blots were hybridized with 5'D9, a part of another desmin clone containing coding sequences $5^{\prime}$ to the position of pDV1 (5'D9 was obtained by digestion of pD9 with Nae I, see Fig. 1), the same single band pattern was generated with BamHI, HindIII, and EcoRI digests as was observed with 3'D8. However, Sac I appears to cleave within an intervening sequence, such that 5'D9 hybridizes only with a region upstream of the intron ( $\approx 3-\mathrm{kb}$ band) while $3^{\prime} \mathrm{D} 8$ hybridizes with a region downstream of it ( $\approx 9-\mathrm{kb}$ band). (The extra weak band in the HindIII digest hybridized with 5' D9 is not the same as that obtained with pD8; the band obtained with 5'D9 is of higher molecular weight and resulted from contamination with pBR322 sequences of the HindIII used to digest the DNA of this blot.) These results suggest that the desmin gene occurs once in the haploid chicken genome. On the other hand, hybridization with another cDNA plasmid isolated using the pDV1 insert as a probe, p50G1 (containing an $\approx 2$-kb insert representing $80 \%$ of the corresponding mRNA, as indicated by RNA blot hybridization data; see below), yielded a pattern shown in Fig. 3. In each of the four digests, a single strong band was observed that corresponds to one of the bands produced by the conserved sequence of $\mathrm{pDV} 1$ or $\mathrm{pD8}$, distinct from the desmin-specific bands. Longer autoradiographic exposure of this blot revealed weak bands that are common to those obtained with $\mathrm{pD} 8$ or $\mathrm{pDV} 1$, corresponding to the desmin-specific bands with which it cross-reacts, as expected. The p50G1 clone was obtained by screening a chicken spinal cord cDNA library with the pDV1 insert. Analysis by two-dimensional gel electrophoresis of the translation products from RNA selected by p50G1 tentatively identified this clone as glial fibrillary acidic protein (GFAP)-specific (results not shown). However, conclusive identification of this probe as GFAP cDNA by nucleotide sequence analysis has not been made. Similar Southern blot analysis using a human type II keratin cDNA plasmid, pKA1 (16), revealed that some of the extra bands that hybridized with pD8 or pDV1 (Fig. 3) possibly result from cross-reaction with type II keratin genes (data not shown).

Nucleotide Sequence Comparison Between Desmin and Other Intermediate Filament Protein Genes. To further explain and confirm the data obtained by the above genomic DNA blot analysis, we compared the desmin nucleotide sequence with those available for mouse GFAP (17), hamster vimentin (18), and human type II keratin (16) cDNAs. Fig. 4 shows part of the results of such analyses. When the first 120 nucleotides of desmin cDNA are compared with the corresponding sequences of the other three cDNAs, $80 \%$ homology between desmin and the three other sequences is found. This region of the gene codes for the most conserved amino acid sequences of the intermediate filament subunits, 58-99 amino acids from the carboxyl terminus (7). Of great interest is the observation that, although chicken desmin, hamster vimentin, mouse GFAP, and human keratin cDNAs exhibit such a high nucleotide sequence homology in this region, the homology between chicken desmin and chicken vimentin cDNAs is much lower, as judged by the absence of crossreactivity in RNA and DNA blot hybridizations. Furthermore, these analyses showed that there is no homology between the different subunits at the $3^{\prime}$ untranslated region of their mRNAs (data not shown).

Expression of the Desmin Gene Is Restricted to Muscle Cells. Total RNA or poly(A) ${ }^{+}$RNA was isolated from tis-

\footnotetext{
D CGACACCTGAAGCATCACATGCCCCCGCACCTGCGCGACTACCAGGACCTGCTCAATCTCAAGATCGCCTTGGACGTCGAGATCGCCACCTACCGCAAGCTGCTCGAGGGCCAGGAGAAC 20

V CAGAACATCAAGGAAGAGATGCCTCGTCACCTTCGTGAATACCAAGACCTCCTCAATCTCAAGATCGCTCTTGACATTGAGATAGCCACCTACAGGAAGCTACTGGAAGCCGAGGAGAGC

G CAAAGCCTCAACGAGGACATCGCCCGCCACCTGCAGGAGTACCAGGATCTACTCAACGTTAAGCTAGCCCTGGACATCGAGATCGCCACCTACAGCAAATTCCTCGAGGGCGAAGAAAAC

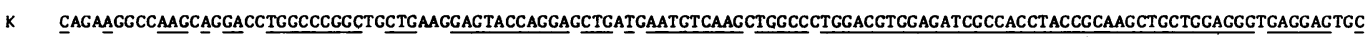

FIG. 4. Highly conserved and variable nucleotide sequences in cDNAs specific for desmin and other intermediate filament proteins. A comparison is shown between the first 120 nucleotides of the chicken desmin cDNA sequence (D) from Fig. 2 and the corresponding sequences of hamster vimentin (V) (18), mouse GFAP (G) (17), and the human epidermal type II 56-kDa keratin KA-1 (K) cDNAs (16). DNA sequences homologous to desmin cDNA are underlined. This highly conserved $(80 \%)$ sequence corresponds to the end of the rod region of the proteins $(7)$. The sequence is shown corresponding to the $5^{\prime}$ to $3^{\prime}$ direction of the mRNA strand. 


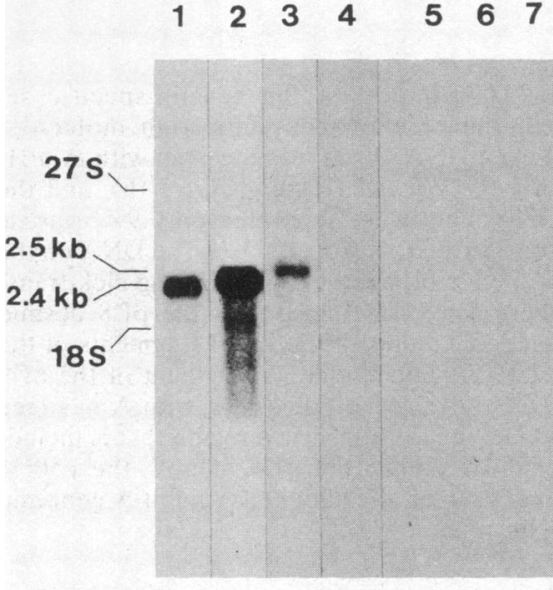

A

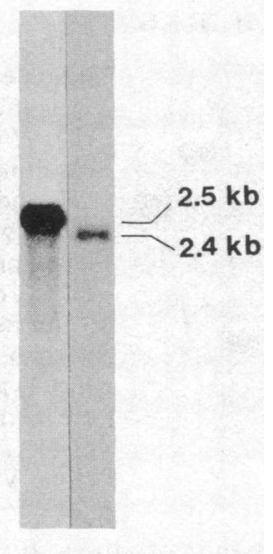

B
Fig. 5. Muscle-specific expression of desmin mRNA. (A) Poly$(\mathrm{A})^{+}$RNA ( $\left.3 \mu \mathrm{g}\right)$ isolated from different chicken tissues as previously described (10) was fractionated on $1.3 \%$ agarose/formaldehyde gels, transferred to nitrocellulose, and hybridized to ${ }^{32} \mathrm{P}$-labeled nick-translated desmin plasmid pDV1. (Identical results were obtained with pD8 or 3'D8.) Lane 1, 1-wk-old skeletal muscle; lane 2, 1-wk-old gizzard smooth muscle; lane 3, 2-wk-old spinal cord; lane 4, 1-wk-old lens; lane 5, 4-day-embryonic erythrocytes; lane 6, 10day-embryonic erythrocytes; lane 7, 15-day-embryonic erythrocytes. (B) RNA blots as in $A$, using $3 \mu \mathrm{g}$ of poly(A) ${ }^{+}$RNA from 2 wk-old spinal cord (lane 1) and 1-wk-old gizzard (lane 2) hybridized with ${ }^{32} \mathrm{P}$-labeled p50G1 cDNA. The positions of the chicken $27 \mathrm{~S}$ and $18 \mathrm{~S}$ rRNA markers were obtained by ethidium bromide staining of total chicken muscle RNA in adjacent lanes.

sues that express desmin, such as gizzard and skeletal muscle, as well as from tissues that, by immunological criteria, do not express it, such as spinal cord, lens, and embryonic erythroid cells. These RNAs were blotted on nitrocellulose filters and hybridized with different nick-translated desmin cDNA probes. From Fig. $5 A$, it is clear that only skeletal muscle and smooth muscle accumulate desmin mRNA and that desmin mRNA appears to be 3-4 times more abundant in gizzard than in skeletal muscle. No detectable desmin se-

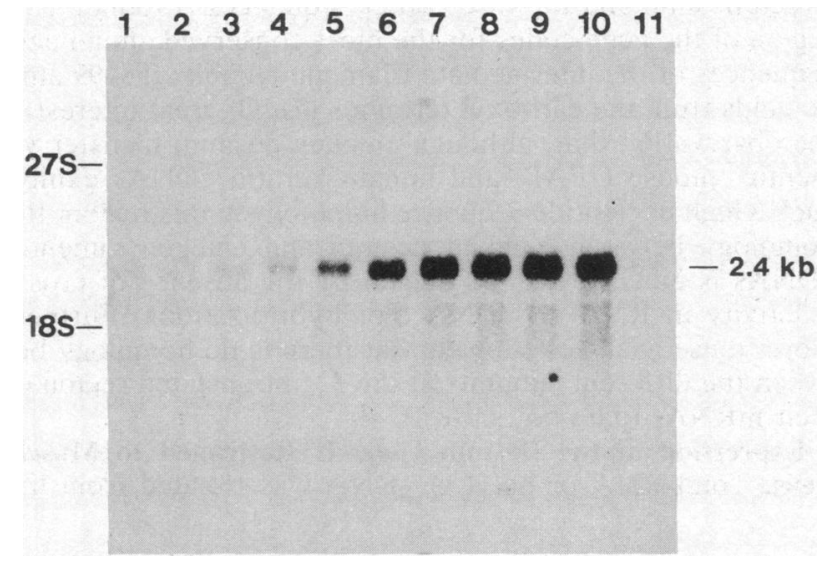

Fig. 6. Developmentally regulated expression of desmin mRNA during myogenesis in vitro. RNA blots similar to those described in Fig. 5 were carried out using $6 \mu \mathrm{g}$ of total RNA from cultured myogenic cells $6 \mathrm{hr}$ (lane 1), $12 \mathrm{hr}$ (lane 2), $24 \mathrm{hr}$ (lane 3), $36 \mathrm{hr}$ (lane 4), 48 $\mathrm{hr}$ (lane 5), $60 \mathrm{hr}$ (lane 6), $84 \mathrm{hr}$ (lane 7), $108 \mathrm{hr}$ (lane 8), $132 \mathrm{hr}$ (lane 9), and $192 \mathrm{hr}$ after plating (lane 10) and from chicken embryo fibroblasts (lane 11). Hybridizations were done with the $\mathrm{pD} 8$ desmin plasmid. Identical results were obtained with the desmin-specific 3'D8 fragment. Marker positions were detected as described in the legend to Fig. 5. quences are observed in lens or erythrocytes of any stage, indicating that the expression of the desmin gene is regulated at the transcriptional level or by mRNA stabilization. In the case of spinal cord, however, a $2.5-\mathrm{kb}$ band is detected when pDV1 or pD8 is used as a probe. This band could represent a higher molecular weight desmin mRNA that is not translated at detectable levels both in vivo and in vitro or it could derive from cross-hybridization of the desmin mRNA with other intermediate filament $\mathrm{mRNAs}$ present in spinal cord, such as those encoding GFAP or the neurofilament $70-\mathrm{kDa}$ subunit. To obtain information on this point, we hybridized the p50G1 cDNA clone described in the previous section with the spinal cord poly $(\mathrm{A})^{+}$RNA. The result of this experiment, shown in Fig. $5 B$, suggests that the desmin-positive signal we obtain with spinal cord RNA (Fig. 5A) arises by cross-reaction of desmin sequences with another putative intermediate filament mRNA (most likely that for GFAP). The converse phenomenon is observed when the p50G1 cDNA is used as probe and cross-hybridization to gizzard desmin mRNA occurs, although to a lesser extent than the corresponding cross-reaction of pDV1 to the presumed GFAP mRNA because there is less sequence homology. When the RNA blot in Fig. $5 A$ was washed and reprobed with the p50G1 cDNA, none of the non-nervous tissues examined displayed detectable amounts of the corresponding mRNA, indicating that the expression of this intermediate filament mRNA is restricted to central nervous tissues. Under the conditions of hybridization used, the p50G1 cDNA cross-reacts only with desmin mRNA and not with vimentin mRNA or any of the neurofilament subunit mRNAs in the spinal cord RNA population. In order to ensure that the patterns obtained by hybridizing skeletal muscle and gizzard RNA with pDV1 or pD8 (Fig. $5 A$ ) reflect the behavior of desmin mRNA and not that of some cross-hybridizing mRNA of the same size, we hybridized similar RNA blots with the desminspecific $3^{\prime} \mathrm{D} 8$ fragment. The results obtained were identical with those of Fig. $5 A$, except that, as expected, no crosshybridization with the spinal cord RNA occurred (data not shown).

Desmin Gene Expression During Myogenesis Is Transcriptionally Regulated. To further investigate the expression of desmin during muscle development, we examined the accumulation of desmin mRNA at various stages of muscle differentiation in tissue culture. Myoblasts analyzed $12 \mathrm{hr}$ after plating exhibit very low levels of desmin mRNA (Fig. 6). Twenty-four to $48 \mathrm{hr}$ after plating, a substantial fraction of the cells have begun to fuse $(\approx 50 \%$ of nuclei are in fused cells at $30 \mathrm{hr}$ ). By $24 \mathrm{hr}$ after plating, desmin mRNA levels increase and continue to do so well after the onset of fusion. Maximal levels of desmin mRNA are observed within 3-4 days after plating and are maintained in the ensuing days of differentiation. No significant further change in the amount of desmin mRNA was observed even 8 days post-plating, at a time that corresponds to the redistribution of desmin to the peripheries of $Z$ discs (3). Small amounts of desmin mRNA were detected also in RNA from nonmyogenic fibroblasts. Similar RNA blot analysis using a vimentin cDNA probe showed constitutive expression of both vimentin mRNAs during myogenesis (10).

\section{DISCUSSION}

Highly Conserved and Variable Nucleotide Sequences Between Desmin and Other Intermediate Filament Proteins. Amino acid sequence data show that the intermediate filament subunits possess a conserved rod domain, with head and tail pieces exhibiting striking divergence $(6,7,16)$. Overall, desmin and vimentin are the most closely related pair of the nonepithelial subunits, sharing $64 \%$ homology over the last 141 carboxyl-terminal amino acids (6). These studies 
compared chicken or mammalian desmin with mammalian vimentin but not chicken vimentin. This is of great importance, because, despite the high amino acid sequence homology between these two proteins, hybridization studies with chicken vimentin probes have not revealed any cross-reaction with other intermediate filament nucleotide sequences $(10,15)$. Furthermore, as we have shown here, the desmin cDNA containing the highly conserved sequences shown in Fig. 4 does not cross-hybridize with RNAs from chicken tissues known to contain vimentin mRNA in high abundance, such as lens, myogenic cells, and embryonic erythrocytes (ref. 10; see also Fig. 5). This indicates that the vimentin gene has diverged from the genes coding for the other intermediate filament proteins to a much greater extent in chickens than it has in mammals. These experiments reveal also that there is a high sequence homology between chicken desmin cDNAs and another putative chicken intermediate filament protein (probably GFAP) cDNA, in contrast to the apparent divergence of chicken vimentin. Furthermore, chicken desmin shares the same highly conserved region with hamster vimentin, mouse GFAP, and human type II epidermal keratins. These highly conserved sequences correspond to the end of coil II of the rod domain (7). Although the chicken vimentin nucleotide sequence for this region is not known, our data indicate that this region of the vimentin gene is much less homologous to the coding sequences for other chicken intermediate filament subunits than the mammalian vimentin gene is to the coding sequences for the other mammalian subunits studied. It seems that selective pressure has either caused a greater divergence of vimentin in chickens from the other subunits or conserved these sequences in mammals.

Tissue-Specific Expression of a Single Desmin Gene. Although no titration experiments have been carried out, the observation of a single band pattern in genomic blots with four different restriction digests and the finding of identical internal restriction fragments in nine desmin cDNA clones suggest that the gene for the intermediate filament subunit desmin occurs only once in the haploid chicken genome; furthermore, we have obtained evidence that the multiple bands obtained in the genomic blots derive at least in part from cross-hybridization of the probes to sequences coding for other members of the intermediate filament superfamily. Another probable single copy gene in chicken appears to be that encoding another intermediate filament protein, most likely GFAP. It has recently been reported that another structurally related intermediate filament subunit, vimentin $(10,15$, $19)$, is also encoded by a single gene in both chickens and hamsters. The accumulation of the desmin protein is tissuespecific and occurs predominantly in smooth, skeletal, and cardiac muscle cells (1). The results presented here indicate clearly that this tissue-specific accumulation of desmin filaments is regulated primarily at the transcriptional or posttranscriptional level. In general, the levels of desmin protein detected previously in smooth and skeletal muscle (5) correlate with the respective levels of desmin mRNA. The observation that very low levels of desmin RNA are present in nonmyogenic fibroblasts grown in tissue culture is in accordance with previous observations showing the presence of low levels of desmin in cultured fibroblasts (3). In chick myogenic cells differentiating in tissue culture, accumulation of desmin mRNA is developmentally regulated and occurs upon fusion to form multinucleate myotubes. In contrast, these cells constitutively express both of the mRNA species encoded by the vimentin gene both before and after fusion (10). Hence, although the desmin and vimentin genes are independently regulated, the expression of these two genes is not mutually exclusive. It appears that functional and morphogenetic requirements dictate the subunit(s) of intermediate filaments that are expressed. Examples of such require- ments may be the involvement of desmin filaments in the lateral linkage of myofibrils to each other and to the plasma membrane at the peripheries of $Z$ discs in muscle cells $(1,4$, 5 ) and the involvement of vimentin filaments in the linkage of the nucleus to the plasma membrane in the nucleated chicken erythrocyte (20). Thus, the accumulation of desmin mRNA during myogenic terminal differentiation is analogous to and concurrent with the accumulation of a number of other mRNAs coding for proteins involved in muscle morphogenesis (21). In conjunction with our previous results on the expression of vimentin during erythroid cell development (10), accumulation of intermediate filaments during terminal differentiation and their tissue-specific subunit composition appear to be regulated primarily at the transcriptional level. Finally, since desmin is probably encoded by a single gene in the haploid chicken genome, and since the levels or size of the single desmin mRNA, as shown in Fig. 6, do not change immediately before, during, or after the onset of the transition of desmin filaments to the peripheries of $Z$ discs, this step in myofibril assembly does not appear to be regulated transcriptionally. The regulation of the distribution of vimentin at this stage of myogenesis also appears not to be regulated transcriptionally (10).

We thank Dr. Richard Ogden for his help in the preparation of the synthetic oligonucleotide probes and Dr. Costantin Flytzanis for his help with the computer sequence studies. We are grateful to Dr. Nicholas Cowan and his colleagues for communicating their data to us prior to publication. We thank Dr. Eric Davidson for his helpful comments on the manuscript. Adriana Cortenbach, Ilga Lielausis, and Susan Stone provided expert technical assistance. This work was supported by grants from the National Institutes of Health, the National Science Foundation, and the Muscular Dystrophy Association of America and by a Grant-in-Aid from the American Heart Association, Greater Los Angeles Affiliate. Y.G.C. was also supported by postdoctoral fellowships from the Muscular Dystrophy Association and from Proctor and Gamble, and J.N., by a Gordon Ross Foundation Predoctoral Fellowship. E.L. is the recipient of a Research Career Development Award from the National Institutes of Health.

1. Lazarides, E. (1980) Nature (London) 283, 249-256.

2. Bennett, G. S., Fellini, S. A., Toyama, Y. \& Holtzer, H. (1979) J. Cell Biol. 82, 577-584.

3. Gard, D. L. \& Lazarides, E. (1980) Cell 19, 263-275.

4. Granger, B. L. \& Lazarides, E. (1978) Cell 15, 1253-1268.

5. Granger, B. L. \& Lazarides, E. (1979) Cell 18, 1053-1063.

6. Geisler, N. \& Weber, K. (1981) Proc. Natl. Acad. Sci. USA 78, 4120-4123.

7. Geisler, N. \& Weber, K. (1983) EMBO J. 2, 2059-2063.

8. Miyoshi, K., Huang, T. \& Hakura, K. (1980) Nucleic Acids Res. 8, 5491-5505.

9. Suzuki, Y., Gage, L. P. \& Brown, D. D. (1972) J. Mol. Biol. 70, 637-649.

10. Capetanaki, Y. G., Ngai, J., Flytzanis, C. N. \& Lazarides, E. (1983) Cell 35, 411-420.

11. Lehrach, H., Diamond, D., Wozney, J. M. \& Boedtker, H. (1977) Biochemistry 16, 4743-4751.

12. Southern, E. M. (1975) J. Mol. Biol. 98, 503-512.

13. Wahl, G. M., Stern, M. \& Stark, G. R. (1979) Proc. Natl. Acad. Sci. USA 76, 3683-3687.

14. Maxam, A. M. \& Gilbert, W. (1980) Methods Enzymol. 65, 499-560.

15. Zehner, Z. E. \& Paterson, B. M. (1983) Proc. Natl. Acad. Sci. USA 80, 911-915.

16. Hanukoglu, I. \& Fuchs, E. (1983) Cell 33, 915-924.

17. Lewis, S. A., Balcarek, J. M., Krek, V., Shelanski, M. \& Cowan, N. J. (1984) Proc. Natl. Acad. Sci. USA 81, 27432746.

18. Quax-Jeuken, Y., Quax, W. \& Bloemendal, H. (1983) Proc. Natl. Acad. Sci. USA 80, 3548-3552.

19. Dodemont, H. J., Soriano, P., Quax, W. J., Ramaekers, F., Lenstra, J. A., Groenen, M. A. M., Bernardi, G. \& Bloemendal, H. (1982) EMBO J. 1, 167-171.

20. Granger, B. L. \& Lazarides, E. (1982) Cell 30, 263-275.

21. Devlin, R. B. \& Emerson, C. P. (1979) Dev. Biol. 69, 202-216. 